\title{
Effect of Braiding Angle on the Impact and Post-Impact Behavior of 3D Braided Composites
}

\author{
S. Yan, ${ }^{1}$ L. Y. Guo, J. Y. Zhao, X. M. Lu, T. Zeng, Y. Guo, and L. Jiang \\ Department of Engineering Mechanics, Harbin University of Science and Technology, Harbin, China \\ 1'yanshi@hrbust.edu.cn (Shi Yan)
}

In this study, the impact and post-impact behavior of three-dimensional (3D) four-directional carbon/epoxy braided composites having different braiding angles were investigated. The same impact energy $(45 . J)$ was applied to the specimens. The post-impact mechanical properties of the materials were performed by compression after impact (CAI) testing, and the processes were monitored by the acoustic emission (AE) technique. Results showed that the specimens with larger braiding angle sustained higher peak loads, and smaller impact damage area, mainly attributed to a more compact space arrangement. The CAI strength and damage mechanism were found to be mainly dependent on the axial support of the braiding fiber tows. Increasing the braiding angle of the composites, the CAI strength was reduced, and the damage mode of the composites was changed from transverse fracture to shear one. Combining AE parameters and CAI curves allows one to characterize the failure process, thereby enabling fracture analysis of the materials under study.

Keywords: braided composites, impact behavior, damage mechanics, acoustic emission.

Introduction. Three-dimensional braided composites have excellent mechanical properties, such as shear strength, resistance impact damage and the structure design, which are widely used in aviation, aerospace and other fields [1-4]. In order to make better use of 3D braided composites, scholars have carried out numerous studies on the relationships between micro- and macromechanical properties, and due to their achievements, the 3D braided composites have gradually became a popular research direction of mechanics, especially relared to the impact behavior [5-10].

Compression after impact (CAI) performance is an important mechanical characteristic for the material design and applications [11]. Therefore, many studies have been carried out to help understanding the CAI response of composite materials and improving the composite design, such as laminates, knitted fabric composites, woven laminates, stitched composites, pyramidal truss core sandwich structures, and so on [12-16]. Compared with normal laminated composites, 3D braided ones offer high impact damage tolerance due to the integrated structure. Consequently, it is important to characterize the CAI performance of $3 \mathrm{D}$ braided composites.

The present work aims to systematically investigate the impact and post-impact responses of braided composite plates. The same impact energy $(45 \mathrm{~J})$ is applied to 3D four-directional braided composites with three different braiding angles. The post-impact mechanical properties of the materials were performed by CAI testing, and the damaged specimens were also visually inspected and monitored by acoustic emission in order to assess the damage mechanism and expanding process.

\section{Experimental.}

1.1. Material Preparation. T700-12K carbon fiber and epoxy resin were used to fabricate the composite specimens through the four-step $1 \times 1$ braiding procedure and resin-transfer molding (RTM) technique. The $120 \times 80 \mathrm{~mm}$ specimens were cut from the flat panels 5-mm thickness. Specimens have similar fiber volume fractions (about 60\%) but different braiding angles $\left(15,25\right.$, and $\left.35^{\circ}\right)$. 
1.2. Impact and CAI Test. In this study, Instron-Dynatup $9250 \mathrm{HV}$ impact testing machine was used according to ASTM D7136. The constant mass of impactor was $7.27 \mathrm{~kg}$ and hemispherical diameter was $15.9 \mathrm{~mm}$. A pneumatic rebound brake was activated to prevent the repeated impact. The same impact energy $45 \mathrm{~J}$ was adopted. At least three specimens were tested for each materials.

INSTRON 3382 test machine (100 kN load cell) was used to test the CAI performances of impacted specimens. Figure 1 shows the CAI test fixture designed and the specimen with strain gauges according to ASTM D7137. The tests were carried out with displacement control, at a rate of $0.5 \mathrm{~mm} / \mathrm{min}$. The CAI strength is calculated by

$$
\sigma^{C A I}=\frac{P_{u}}{b t}
$$

where $\sigma^{C A I}$ is the CAI strength, $P_{u}$ is the ultimate compressive load, $b$ is the average width, and $t$ is the average thickness.
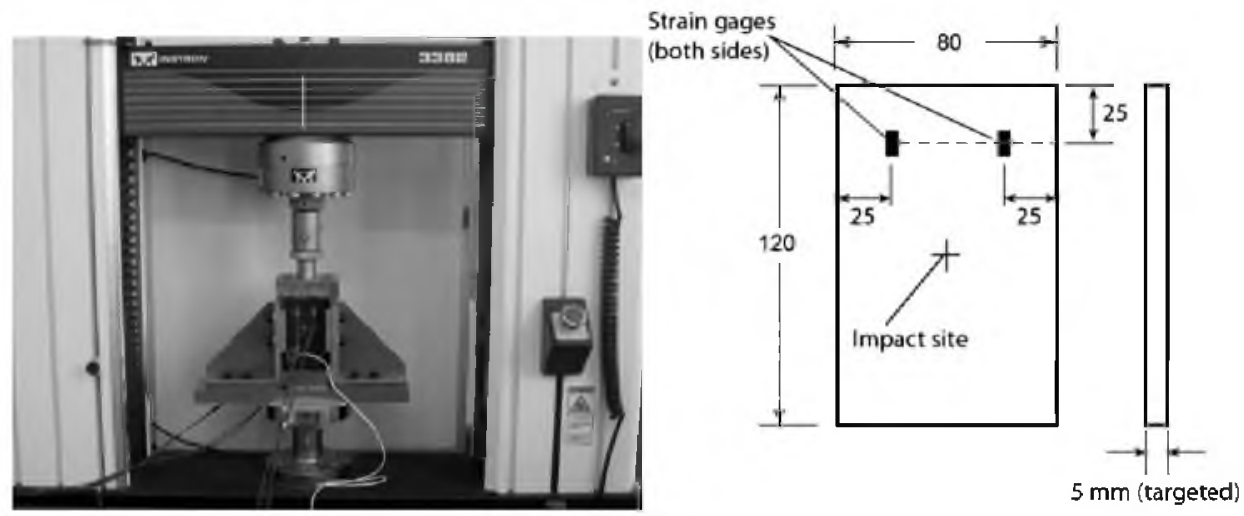

Fig. 1. Compression after impact setup and CAI specimen with strain gauges.

Calculation of the effective compressive modulus using Eq. (2), and the applied force at 1000 and 3000 microstrain is based on the average strain for all four strain gauges

$$
E^{C A I}=\frac{P_{3000}-P_{1000}}{\left(\varepsilon_{3000}-\varepsilon_{1000}\right) b t},
$$

where $E^{C A I}$ is the CAI modulus.

In this work, a four channel acoustic emission (AE) system, supplied by Physical Acoustics Corporation (PAC) was used to record the AE signals. AE measurements were achieved using a broadband PAC sensor with the frequency range of $100-1000 \mathrm{kHz}$. Pre-amplification of $40 \mathrm{~dB}$ and threshold of $45 \mathrm{~dB}$ were performed.

\section{Results and Discussion.}

2.1. Impact Tests. The resulting parameters are summarized in Table 1. The integrated structures get more compact with the braiding angle, and then provide the limited impact damage extension and improve the shock resistance of composites, leading to higher peak loads and smaller impact damage areas.

There are some oscillations in the unloading regions (Fig. 2) induced by breakage of face sheet directly under the impact region, and the braided nature of the materials offers a significant resistance to the breakage of fibers. 
S. Yan, L. Y Guo, J. Y. Zhao, et al.

$\mathrm{T}$ a b 1 e 1

Parameters of 3D Braided Composites Impacted by $45 \mathrm{~J}$

\begin{tabular}{|c|c|c|c|c|c|}
\hline Type & $\begin{array}{c}\text { Braiding } \\
\text { angle } \\
(\mathrm{deg})\end{array}$ & $\begin{array}{c}\text { Impact } \\
\text { energy } \\
(\mathrm{J})\end{array}$ & $\begin{array}{c}\text { Absorbed } \\
\text { energy } \\
(\mathbf{J})\end{array}$ & $\begin{array}{c}\text { Peak load } \\
(\mathrm{kN})\end{array}$ & $\begin{array}{c}\text { Deflection } \\
\text { at peak load } \\
(\mathrm{mm})\end{array}$ \\
\hline I & 15 & 44.07 & 42.10 & 10.27 & 5.98 \\
\hline II & 25 & 43.32 & 41.48 & 11.02 & 5.46 \\
\hline III & 35 & 43.45 & 41.78 & 12.30 & 5.10 \\
\hline
\end{tabular}

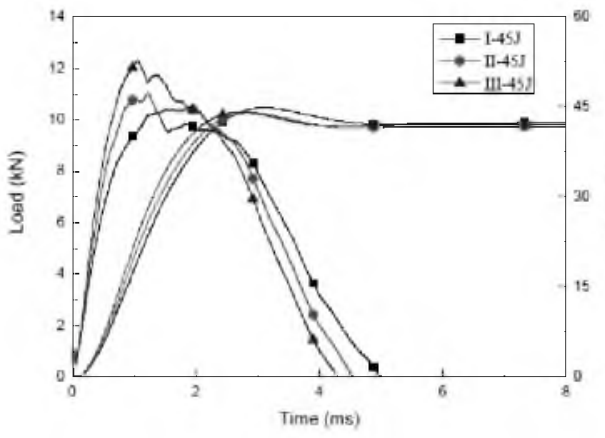

a

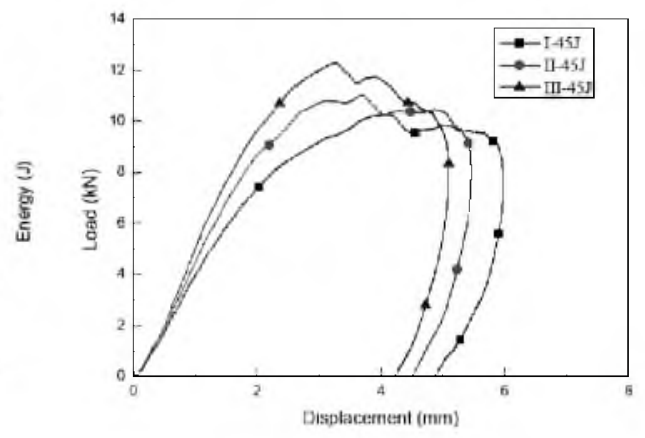

b

Fig. 2. Representative curves from the impact tests: (a) load and energy versus time curves; (b) load versus displacement curves.
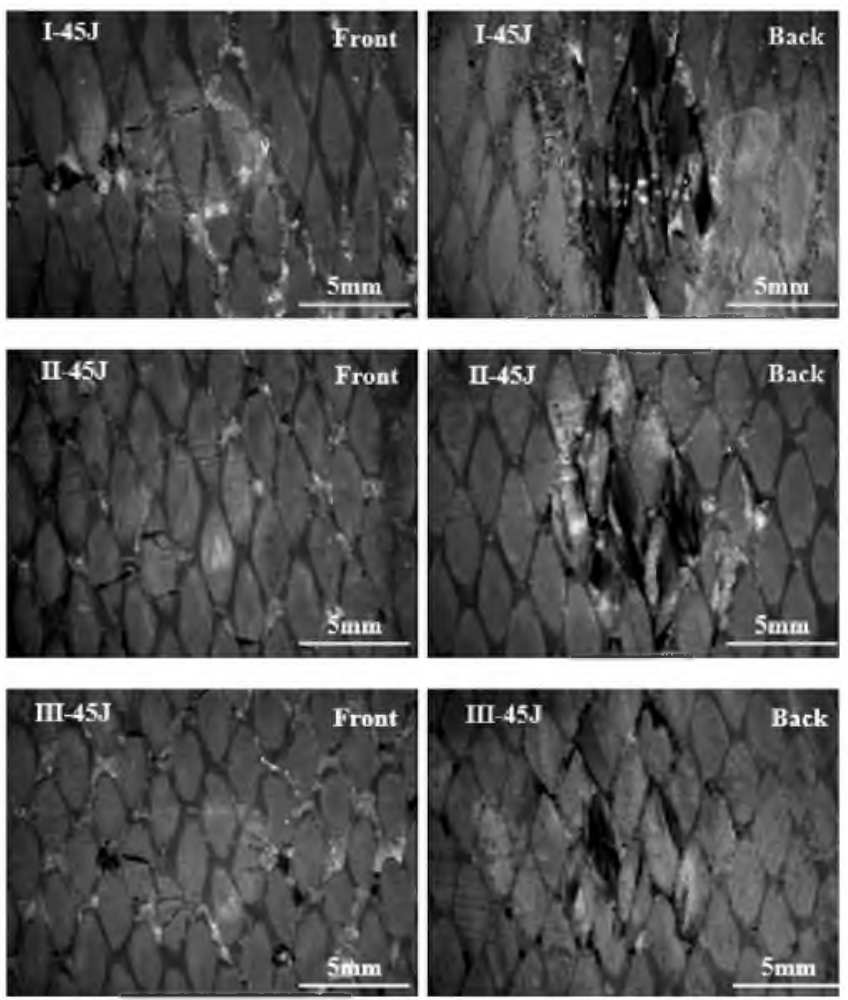

Fig. 3. Images of damaged specimens impacted by $45 \mathrm{~J}$. 
Figure 3 shows the damaged specimens after the impact testing. There is the back surface-splitting, while fiber breakage appears at the back surface, which means the interior fiber is broken in the impact area. The damaged area was much larger in specimens with smaller braiding angles, due to the respective density of the fiber tow arrangements. For the 3D braided composites, an increase in the braiding angle results in a more compact arrangement of the fiber tows, limited damage extension, and improved shock resistance of the composite.

2.2. CAI Tests. Typical compressive curves of the specimens are presented in Fig. 4. For the 15 and $25^{\circ}$ specimens, the curves follow a linear (elastic) pattern, and then exhibit a sharp drop as the result of brittle fracture. However, for the $35^{\circ}$ specimens, the curve has a nonlinear tendency versus load. This indicates that the CAI strength decreases with the braided angle (Fig. 5a), which can be attributed to the following two factors. Firstly, the axial support of the braiding fiber tows is the dominating bearing capacity. The smaller braiding angle specimens can withstand higher axial loads under the same compressive load conditions, and the axial direction mainly coincides with the loading direction. Secondly, the fracture/damage modes are different, which issue will be discussed in more detail in the next section. The compressive modulus of 3D braided composites has a similar variation trend at the CAI strength (Fig. 5b).

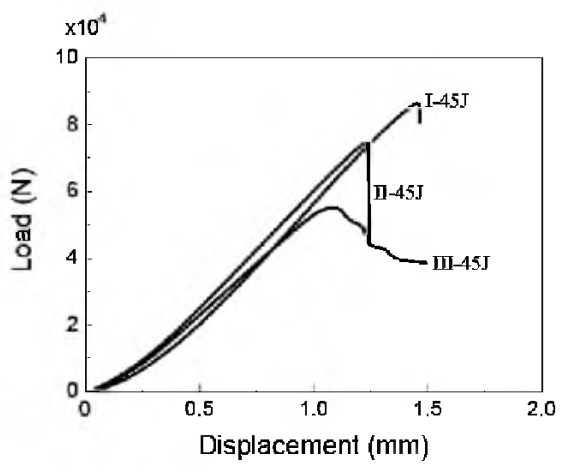

Fig. 4. CAI response curves of specimens.

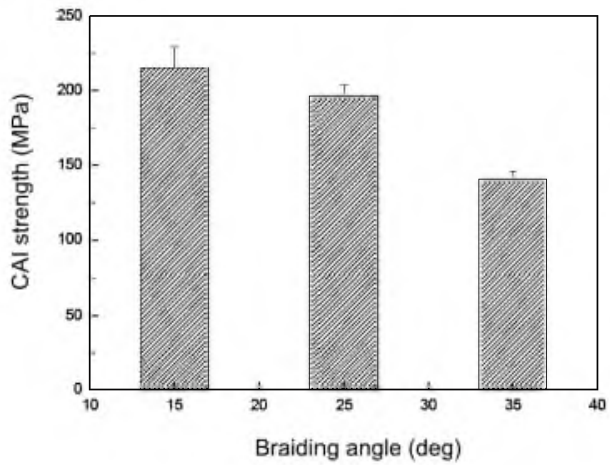

a

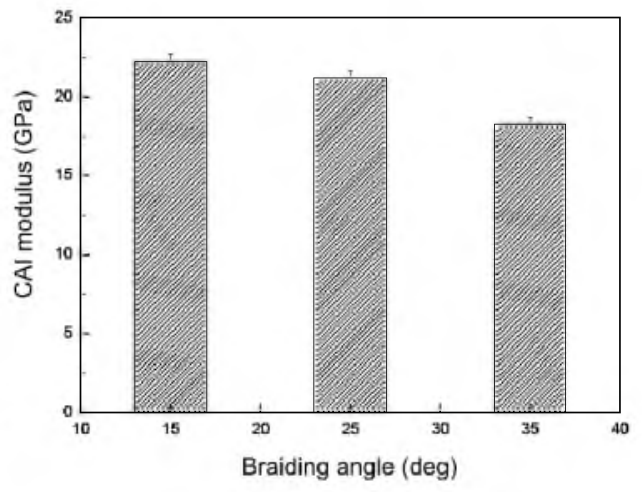

b

Fig. 5. CAI test results: (a) CAI strength and (b) CAI modulus.

Figure 6 displays detailed fractographies of CAI specimens. For the 15 and $25^{\circ}$ specimens, fiber breakage occurs in numerous fiber tows, and then the total structure 

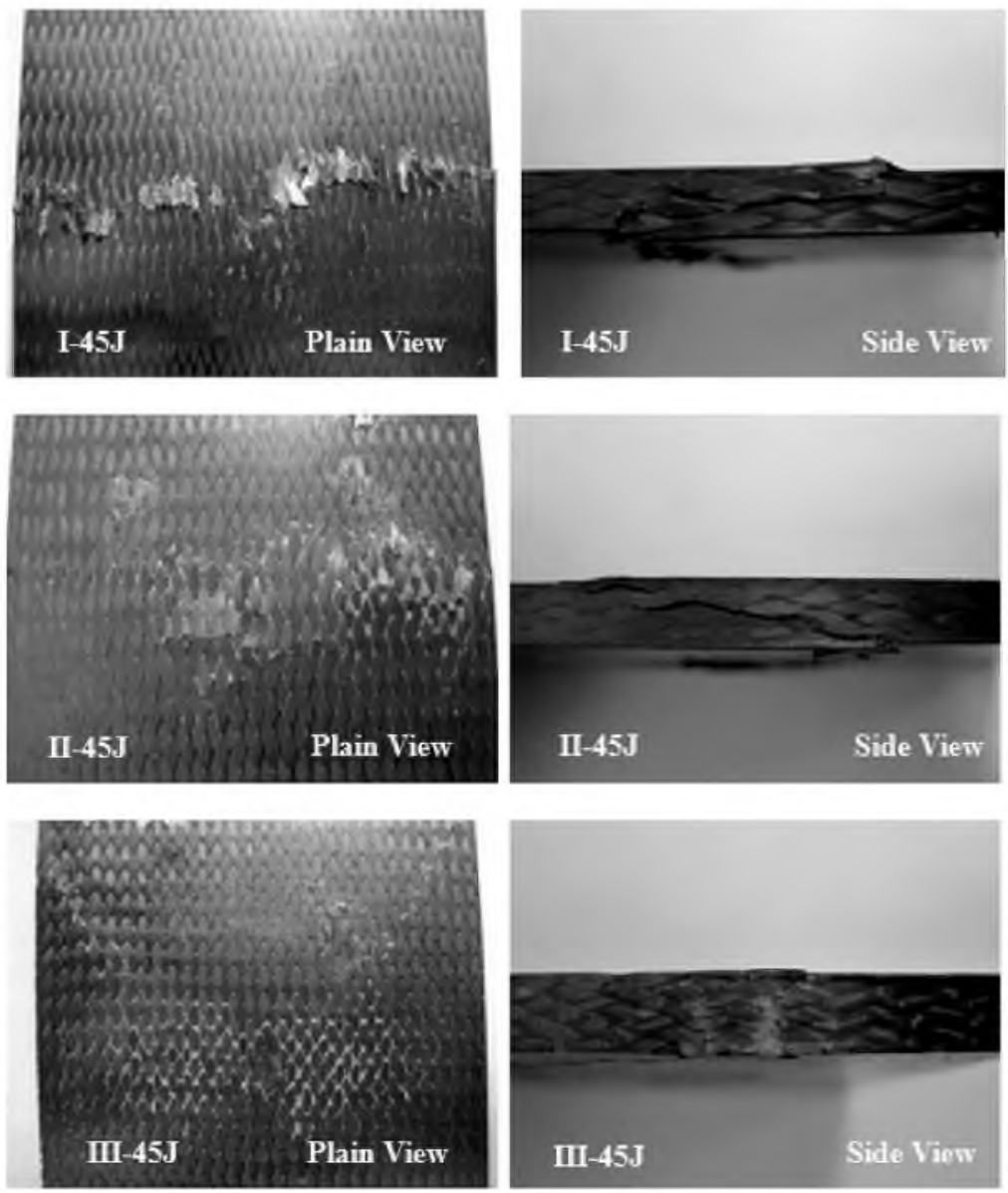

Fig. 6. Typical failure photos for CAI specimens.

undergoes the brittle fracture. Here the fiber tows breakage along the braiding angle can be observed from the side view. For the $35^{\circ}$ specimens, the main failure mode is the composite shear fracture along the braiding direction reaching the specimen boundary.

The different CAI failure modes resulted from the braiding angle variation. As for the smaller braiding angle specimen, the fiber tows were closer to the compressive direction, resulting in the compression process, the fiber tows could withstand higher loads, thus enhancing the specimen bearing capacity. When reaching the limit load, fiber tows are prone to brittle breakage, and the specimens were fractured. With an increase in the braiding angle, the matrix sustained higher loads, the matrix crack and interface debonding happened before the fiber breakage, and then the shear failure occurred along the braiding direction without the support of the matrix. So the CAI strength of $35^{\circ}$ specimens is lower than that of other specimens, in spite of a smaller impact damage under the same impact energy level, and the load-displacement curves show a nonlinear tendency with no sharp drops after application of critical compressive load.

2.3. AE Behavior and Characterization. Based on the characterization of $\mathrm{AE}$ amplitude [17] and normalized cumulative AE energy [18], the CAI failure mechanisms and process were assessed. The representative curves of load and AE amplitude vs. displacement for the specimens in Fig. 7. To investigate the CAI failure process of the composites, the response of normalized cumulative AE energy is shown in Fig. 8. The 


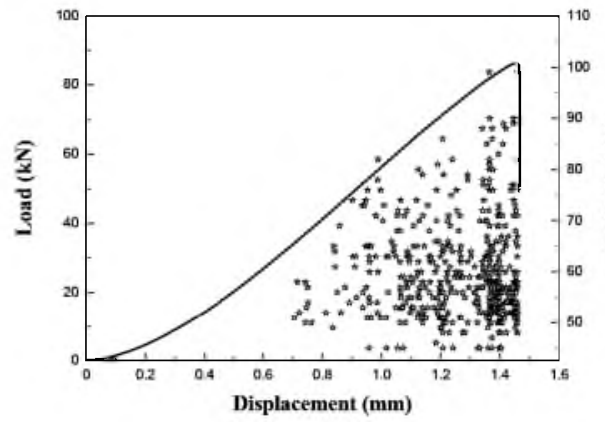

a

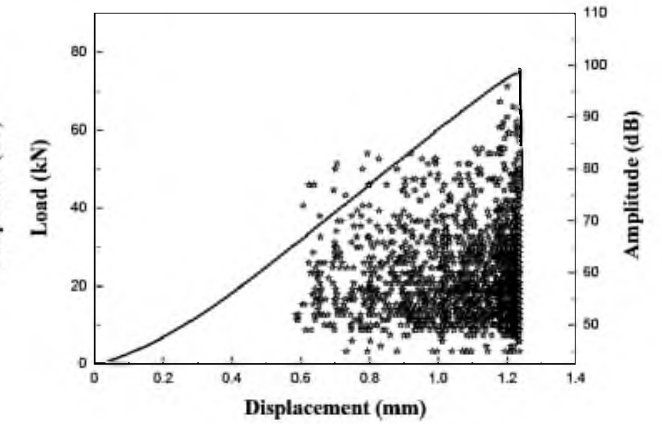

b

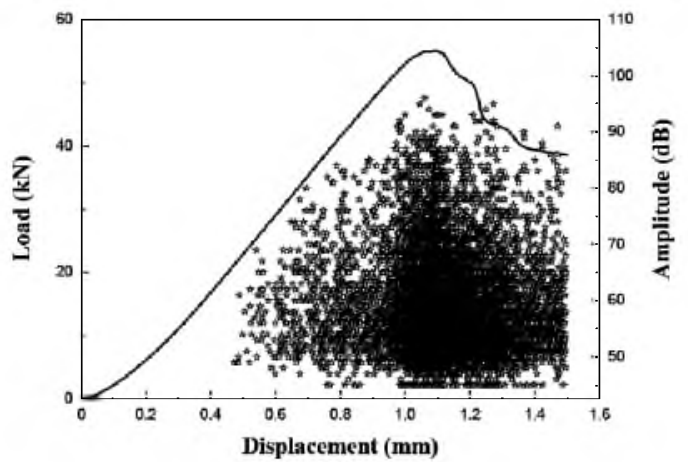

$\mathrm{c}$

Fig. 7. Typical load and AE amplitude vs. displacement curves for the specimens: 15 (a), 25 (b), and $35^{\circ}$ (c).

normalized cumulative AE energy is defined as the total cumulative AE energy divided by the sum of individual $\mathrm{AE}$ energies,

$$
\text { Normalized cumulative } \mathrm{AE} \text { energy }=\sum_{i=1}^{a} E_{i} / \sum_{i=1}^{n} E_{i} \quad(n \geq a),
$$

where $n$ is the number of total AE event and $a$ is the number of $i$ th $\mathrm{AE}$ event.

In Fig. $8 \mathrm{a}$ and $\mathrm{b}$, the normalized cumulative $\mathrm{AE}$ energy sustained to augment before the $90 \%$ maximum load, while the AE amplitudes with $50-80 \mathrm{~dB}$ are depicted in Fig. $7 \mathrm{a}$ and $\mathbf{b}$. These $\mathrm{AE}$ features suggest that the propagation and extension of impact damage is accompanied by fiber-matrix debonding and fiber pull-out. Thereafter, the slope of cumulative AE energy showed an obvious increase, and some high amplitude signals over $85 \mathrm{~dB}$ appeared, which indicates another failure mechanism such as fiber breakage occurred. And then an AE hit event with the highest energy began to appear at the ultimate load, and the slope of cumulative AE energy was greatest. But the magnitude of changes meant different fail mechanisms. For the 15 and $25^{\circ}$ specimens, as shown in Fig. 8a and b, the cumulative AE energy was suddenly increased, which is attributed to fiber tows' breakage and matrix fracture at the same time under ultimate load, a large elastic energy released as a strong $\mathrm{AE}$ signal with high $\mathrm{AE}$ amplitude and $\mathrm{AE}$ energy, and the blasting sound of brittle fracture was recorded. For the $35^{\circ}$ specimen, as shown in Fig. 8c, the cumulative AE energy did not exhibit any obvious increase. It means that the AE energy level of $35^{\circ}$ specimen is much lower than those of other specimens. The elastic energy released as a weaker AE signal with high amplitude and low energy, as the result of the 


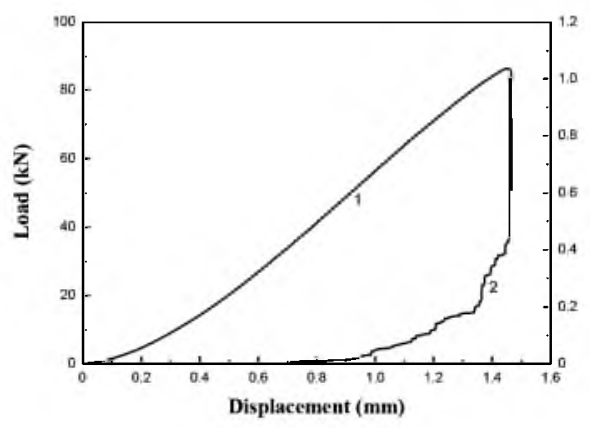

a

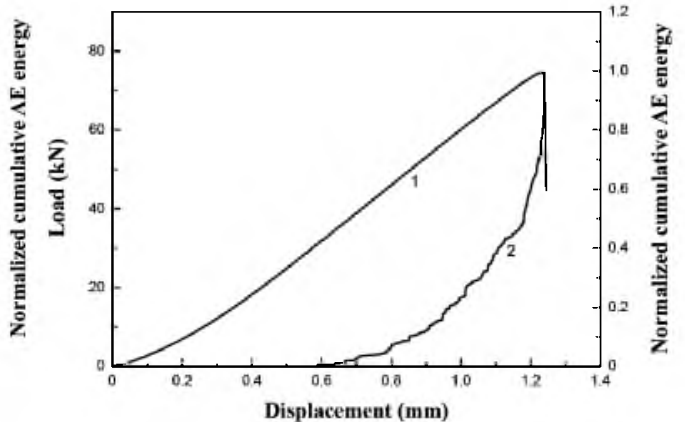

b

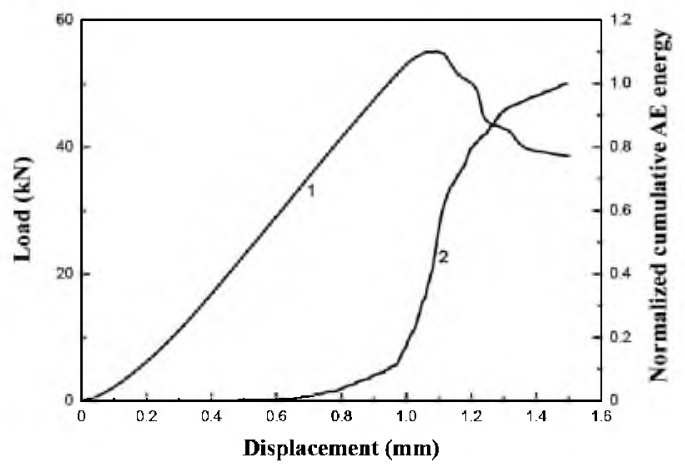

$\mathrm{C}$

Fig. 8. Typical load-displacement curves (1) and normalized cumulative AE energy vs. displacement curves (2) for the specimens with different braiding angles: 15 (a), 25 (b), and $35^{\circ}$ (c).

fiber damage due to the fiber pull-out and yarn-to-yarn friction during the specimen shear failure. Therefore, the cumulative AE energy sustained to augment but the slope showed a gradual decrease. The amplitude distributions (Fig. 7c) fluctuated between 50 and $95 \mathrm{~dB}$, which amplitude levels can be attributed to fiber-matrix debonding, fiber pull-out and yarn-to-yarn friction. Conscquently, the CAI damage process in materials can be described by the $A E$ features.

Conclusions. The effect of braiding angle on the low-velocity impact and compression after impact characteristic of 3D four-directional braided composites were experimental investigated. The following conclusions can be drawn from the results:

1. For the $3 \mathrm{D}$ braided composites, an increase in the braiding angle results in a more compact arrangement of the fiber tows, limited damage extension, and improved shock resistance of the composite, leading to higher peak loads and smaller impact damage areas.

2. CAI results indicate that smaller braiding angle specimens have higher CAI strength and elastic modulus, which is mainly attributed to the axial sustain of braiding fiber tows. The braiding angle variation evokes different CAI failure modes. As specimens with smaller braiding angles, fiber tows brittle breakage lead to transverse fracture of the compositc. With increased braiding angle, the shear failure of the specimen occurs along the braiding direction.

3. It is confirmed that $\mathrm{AE}$ is an effective tool to acquire in situ information during the failure process on the CAI tests. The failure process of the materials showed multiple damage mechanisms, such as matrix crack, fiber-matrix debonding, fiber pull-out, yarn-toyarn friction and fiber breakage, while the failure mechanisms have obvious distinctions for the specimens with different braiding angles. 
Acknowledgments. The authors would like to thank the National Natural Science Foundation of China $(11102055,11272110)$, the Science and Technology Innovation Team of Heilongjiang Department of Education (12521102).

1. T.-W. Chou and F. K. Ko, Textile Structural Composites, Elsevier, Amsterdam (1989).

2. L. Chen, X. M. Tao, and C. L. Choy, "On the microstructure of three-dimensional braided performs," Compos. Sci. Technol., 59, 391-404 (1999).

3. S. Abrate, Impact on Composite Structures, Cambridge University Press (1998).

4. M. Shake, F. Ko, and J. Song, "Comparison of the low and high velocity impact response of Kevlar fiber-reinforced epoxy composites," J. Compos. Tech. Res., 21, 224-229 (1999).

5. T. Zeng, D. N. Fang, and T. J. Lu, "Dynamic crashing and impact energy absorption of 3D braided composite tubes," Mater. Lett., 59, 1491-1496 (2005).

6. J. D. Littell, W. K. Binienda, W. A. Arnold, et al., "Effect of microscopic damage events on static and ballistic impact strength of triaxial braid composites," Compos. Part A-Appl. S., 40, 1846-1862 (2009).

7. B. H. Gu and J. G. Xu, "Finite element calculation of 4-step 3-dimensional braided composite under ballistic perforation," Compos. Part B-Eng., 35, 291-297 (2004).

8. B. Z. Sun and B. H. Gu, "High strain rate behavior of 4-step 3D braided composites under compressive failure," J. Mater. Sci., 42, 2463-2470 (2007).

9. Y. Zhang, B. Z. Sun, and B. H. Gu, "Experimental characterization of transverse impact behaviors of four-step 3-D rectangular braided composites," $J$ Compos. Mater., 46, No. 24, 3017-3029 (2012).

10. M. Dale, B. A. Acha, and L. A. Carlsson, "Low velocity impact and compression after impact characterization of woven carbon/vinylester at dry and water saturated conditions," Compos. Struct., 94, 1582-1589 (2012).

11. H. Yan, C. Oskay, A. Krishnan, and L. R. Xu, "Compression-after-impact response of woven fiber-reinforced composites," Compos. Sci. Technol., 70, No. 14, 2128-2136 (2010).

12. G. Minak and D. Ghelli, "Low velocity impact and compressive after impact tests on thin carbon/epoxy laminates," Compos. Part B-Eng., 42, 2067-2079 (2011).

13. K. T. Tan, N. Watanabe, Y. Iwahori, and T. Ishikawa, "Effect of stitch density and stitch thread thickness on compression after impact strength and response of stitched composites," Compos. Sci. Technol., 70, 587-598 (2012).

14. G. Q. Zhang, B. Wang, L. Ma, et al., "The residual compressive strength of impact-damaged sandwich structures with pyramidal truss cores," Compos. Struct., 105, 188-198 (2013).

15. A. Aktas, M. Tercan, M. Aktas, and F. Turan, "Investigation of knitting architecture on the impact behavior of glass/epoxy composites," Compos. Part B-Eng., 46, 81-90 (2013).

16. B. Vieille, V. M. Casado, and C. Bouvet, "Influence of matrix toughness and ductility on the compression-after-impact behavior of woven-ply thermoplastic- and thermosettingcomposites: A comparative study," Compos. Struct., 110, 207-218 (2014).

17. X. M. Zhuang and X. Yan, "Investigation of damage mechanisms in self-reinforced polyethylene composites by acoustic emission," Compos. Sci. Tech., 66, 444-449 (2006).

18. S. C. Woo and T. W. Kim, "High-strain-rate impact in Kevlar-woven composites and fracture analysis using acoustic emission," Compos. Part B-Eng., 60, 125-136 (2014). 